\title{
Based on asynchronous communication protocol of geographic space information service access mechanism research
}

\author{
Chen Guo-ping ${ }^{\mathrm{a}, \mathrm{b}}$, Zhao Jun-san ${ }^{\mathrm{a}, \mathrm{c}}, \mathrm{Gu}-\mathrm{Miao}{ }^{\mathrm{c}}$, Li Dong-sheng ${ }^{\mathrm{b}}$ \\ (a .Faculty of Land Resource Engineering, Kunming University of Science and Technology, Kunming

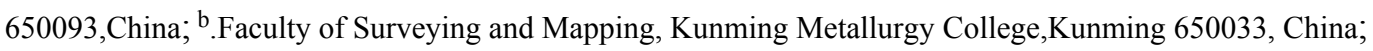 \\ ${ }^{\mathrm{c}}$.Kunming Yunjindi Geo-Information Co.Ltd.,Kunming 650106, China)
}

Keywords: geographic spatial information services, Asynchronous transmission mechanism, Location service

\begin{abstract}
:
At present, the traditional way of accessing to classified network in geographic spatial information services is using network gatekeeper and firewall etc. to ensure public and classified network communications links. However, the physical isolation between classified network and public network is crossed, which is bound to cause classified network potential security hazard. In Yunnan province space Land dynamic monitoring integration project, it proposed the point to point text message communication protocol and asynchronous transmission mechanism. Using geo-spatial information encryption processing and data compression processing method, it reduced the risk of data sensitivity and monitored, namely to ensure data security, which realized geographic spatial information services data communication effectively between classified network and public network in the rigid field conditions.
\end{abstract}

\section{INTRODUCTION:}

In the process of land and resources law enforcement is commonly in the field, but it needs analyze and the query a large number of geo-spatial data which saved in classified network. Provided it accessing directly, it needs break through the physical isolation between the classified network and the network. Traditional access method using GPRS and $3 \mathrm{G}$ network, which expose the server to the Internet environment.Although network gatekeeper and firewall are used, the physical isolation between classified network and public network is crossed, which is bound to cause classified network potential security hazard. Besides, land and resources Law enforcement is mainly in the suburbs and field, where $3 \mathrm{G}$ network is not covered completely, some location, even GPRS network communication, cannot be guaranteed. Land and resources law enforcement Chen Guo-ping email: 115432640@qq.com urgently need a new kind of methodology to tackle the problem of accessing geo-spatial data between the classified network and the network.

SMS (short message service) is a store and forward service, first of all, the initiator terminal sends data to the server transitorily; After that the server store and forward data; Finally, the receiver terminal passive receiving data from the server with unpersistent connections way, the receiver terminal does not send feedback information to the initiator. This method is not easy to be sniffed and monitored by the third party. In addition, the requirement of this way for network environment is not strict, as long as to meet the requirements of digital voice communication, it can conduct the service. And communication cost is low. But the SMS service has a data transmission size limit every time that means every time transfer shall not exceed 70 characters or 140 bytes. There are massive number 
of research on small amount of data transmission monitoring indicators such as low, pressure, etc. in domestic. However, as to large amount of data transmission investigation, like geographical spatial information query, is rare.

The author taking Yunnan province space Land dynamic monitoring integration project as instance, proposed asynchronous communication protocol based on SMS service, using coordinate offset and data compression method to fulfill geo-spatial information accessing service in the stringent conditions.

\section{DATA COMMUNICATION PROTOCOL}

Through the investigation of land and resources law enforcement business process, we found that the wild enforcement is mainly though the inspectors to carry terminal equipment with data acquisition functions collecting geo-spatial information. Then overlapping collecting geo-spatial information with existed graphic data that is classified. After that inspectors analyze various index to make a judgment whether collecting geo-spatial data from the reality environment just now satisfy existed graphic data requirement. Due to security requirements, field inspectors cannot carry large amounts of confidential graphic data. Field inspectors submit the collected geographic spatial information data to the server, the server receive the data and analyze it. Then the analysis results are returned to the field inspectors, field inspectors acquire information of if the land use is illegal. In order to guarantee the security, terminal equipment and the server shall encipher the information before sending. When the receiver can decipher the encode information the data dictionary which promissory in advance. Program flow as Figure 1.

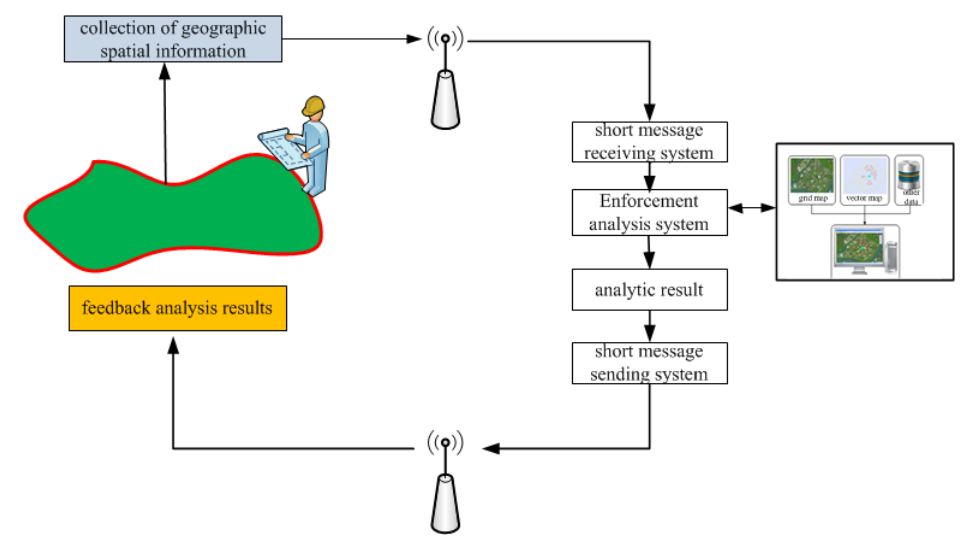

Figure 1. Program flow

\section{1 data structure}

Through the analysis of data transmission, transmitted data content two types, one is geographical spatial coordinate information which terminal equipment submitted to the server, the data structure is regular, format-fix; the other is the analysis results that the server returned to the terminal equipment. This type data is text information, the length of data is flexible. The difference of the two types of data structure is large, it is necessary to design a common data structure to carry the query information. The primary data structure design of the whole system is shown in Figure 2.

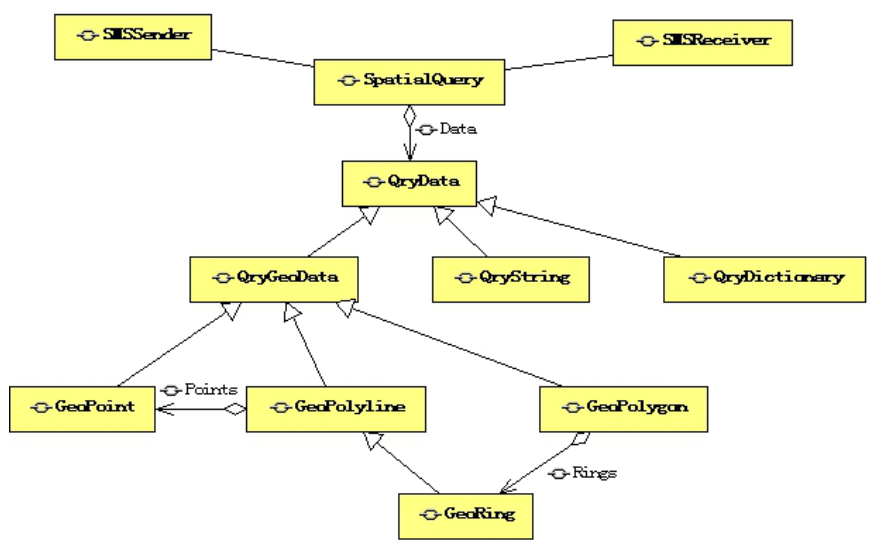

Figure 2 data structure

System packaged all needed data transfer request as SpatialQuery,the statement is as follows:

public class SpatialQuery\{ private QryData data $=$ null; private int queryType $=-1$; public QryData getData()\{return data; 
public int getQueryType() \{return queryType;

\} ;

The queryType defines the types of queries, through code it distinguishes data is query requests or feedback the result QryData class is base class of all request and response data type. QryData class is an abstract class, considering the data is taken mainly by the form of text messages, so the corresponding query data value needs be converted into a string:

public abstract class QryData \{ public abstract string ToString();

\}

QryData has three implementation class, namely QryString, QryDictionary and QryGeoData, the QryString is responsible for data string type exchange, which used for returning query results ; And QryDictionary is used in key-value type data exchange, which can achieve more parameters query and the more result data return; QryGeoData is an abstract class, it is the abstraction of geographical spatial query object, which is divided in to GeoPoint, GeoPolyline and GeoPolygon three implementation class according to the different types of terrain. GeoPoint class save a coordinate information, including the points longitude and latitude coordinates; A butch of points coordinate with the order connected together is a line, namely GeoPolyline class; GeoRing is a special GeoPolyline, whose fore and rear is the same point; Multiple GeoRing constitutes the GeoPolygon, it represented the island and hole of Polygon by GeoRing rotation direction, clockwise Ring is island, counter-clockwise Ring is hole. Each type of GeoData contains a large number of data points coordinates.

\section{2 transmission optimization}

Considering limitation length of data for the message communication protocol each time, we design the coordination offset and data compression to reduce the length of the data transmission. The two optimization scheme improve the transmission efficiency and reduce the transport cost.

\subsubsection{Coordinate offset}

Geo-spatial data transmission is mainly coordinate point transmission, considering information transmission security, with longitude and latitude method for data transmission. Because of higher accuracy of latitude and longitude coordinates, the meters-level accuracy data needs seven decimal places generally. If it store the information with character type, a number will reach 11 byte, a coordinate point account at least 22 byte, so a text message can carry little information. Considering the geographic space information query is usually no more than several kilometers, we adopt coordinates offset to shorten the length of coordinates: if needed transmitted data contain multiple points (for a single point, this way is no value), it amplify all latitude and longitude coordinates to integer. Completing data unit conversion, it selects minimum longitude and latitude in the all points as a relative coordinate system origin, so all of the data converted into positive integer, which achieve the purpose of using short integer to indicate the floating point coordinates. The example is shown in figure 3 .

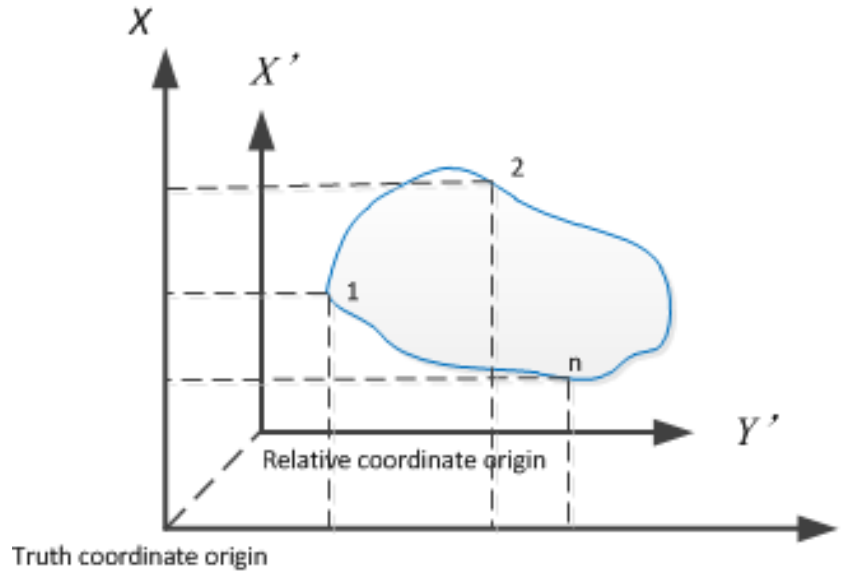

Figure 3 coordinate offset

Space coordinate representation type is: (the dot number, the longitude, the latitude). If a graphics, the real coordinate system is expressed as $(1, \mathrm{XXX}$. 
$* * * * 111, \mathrm{yy} . * * * * 111),(2, \mathrm{XXX} . * * * * 222$, yy. $* * * * 222), \ldots(\mathrm{n}, \mathrm{XXX} . * * * * \mathrm{NNN}, \mathrm{yy} . * * *$ *]). Using coordinate offset processing methods mentioned above, the relative coordinate system is expressed as (1', 111111), (2', 222222),... (n, NNN, NNN). During transmission, it only transfers offset value between original coordinates system and offsetting coordinates system, and converted relative coordinates string. The efficiency of this method as to single point coordinate data tis not obvious, but for multiple-point data transmission efficiency is remarkable, it can improve transmission efficiency greatly.

\subsubsection{Data compression}

Considering the cost, as to large amount of data it need use a data compression method to reduce amount of data transmission. With the compressed content, GeoString and GeoDictionary objects, the amount of the transmission data is unpredictable, so LZW compression (Lempel - Ziv - Welch Encoding, LZW) is used to compress dynamic string coding.

The principle of LZW compression algorithm ${ }^{[5]}$ is to extract different characters of the original text file data and to create a table on the basis of these characters. Then use the index of the characters compile table instead of the corresponding characters in original text file data, which reduces original data size. But the compile table is not to create in advance, it will be created according to the original file data dynamically. When decoding it restore the original text file data from the encoded data compilation tables. The description is shown in Figure 4.

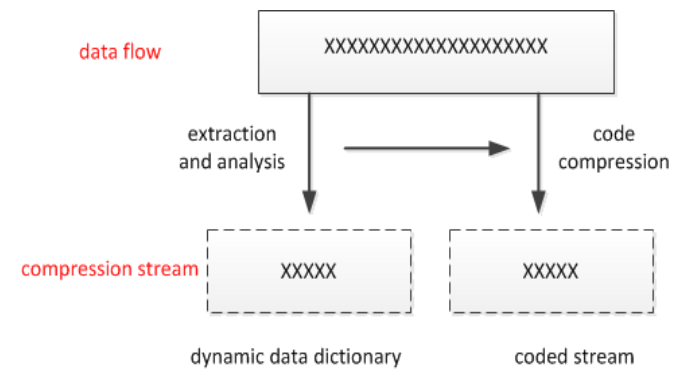

Figure 4 LZW compression algorithm
As to GeoData object, coordinate data is transmitted, content is digital character (" 0 ", "9") and the space character (", "and"; "), the scope of the characters is fixed, so using a LZ77 algorithm ${ }^{[6]}$ of fixed dictionary tables compress the data.The example is shown in Figure 5.

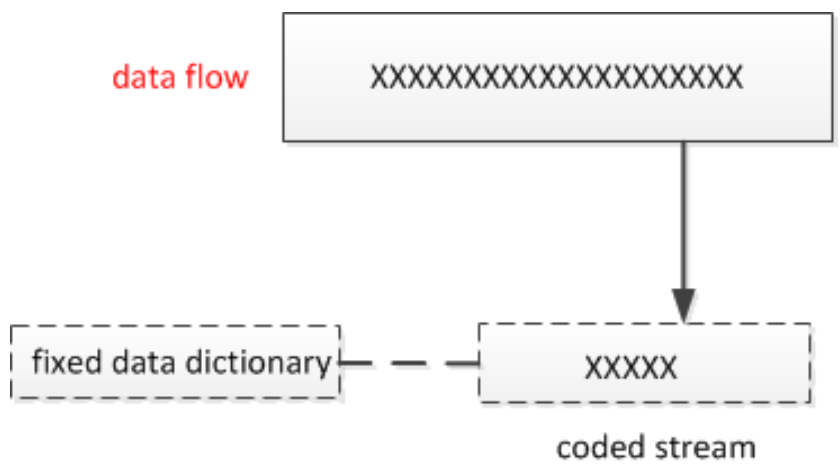

Figure 5 LZ77 algorithm of fixed dictionary tables compress algorithm

\section{ASYNCHRONOUS TRANSMISSION MECHANISM AND IMPLEMENT}

In SMS, Sender is a class which is charge of sending text messages in the system, requiring sending data (query requests and feedback result) need paging processing according to data size. If sending data amount is not more than 140 bytes, then send it directly. If sending data amount more than 140 bytes, it needs paging processing when the terminal receiver splice paging data automatically. Of course some communication service providers support to the large data amount sending functions (more than 140 bytes of text message), which sends more than 140 bytes of information directly. But most of the communications service do not provide this large data amount sending function. Therefore, considering the compatibility of the system application, SMS Sender made paging treatment to more than 140 bytes data uniformly

In SMS Sender received the query information request, then it will perform paging processing according to the query information paging size, besides it adds query information identification, the total number of paging, the current paging number 
three parts information on each page of head, before sending data. The paging structure description is as follows:

\section{SMSMessage $=$ record} integer id; //query information identification integer Count; //total number of paging integer Index;//current paging number char[ ] Data;

end;

In SMS receiver is responsible for receiving messages. Due to the short message communication protocol is an asynchronous communication, the sender submitted the data to the server, the server sending data, which does not guarantee that the receiver receive the data with the order of sending. it needs receiver analyse the data content, whether text messages have contains all the data which sends by server, if it does not, the data already received is written to temporary files, until all data is received, the receiver assemble data content with the order of Index; If it has obtained all the data, then it analyzes data and creates the corresponding query object (SpatialQuery), conducting query analysis work.

\section{CONCLUSIONS AND PROSPECT}

With the strict data confidentiality requirement, rigid condition demand of field data transmission, it proposed point to point communication protocol based on SMS service, relying on data asynchronous transmission mechanism and data compression method, which achieved accessing of geographic spatial information services in classified the network in field. The author taking Yunnan province space Land dynamic monitoring integration project as experiment, the results show that the asynchronous communication mechanism can improve the inspect data analysis efficiency of land and resources law enforcement in field greatly, which provides way for using confidential information of land and resources reasonably.

\section{ACKNOWLEDGMENT}

References from websites:

Baike[EB/OL].http://baike.baidu.com/subview/4427

6/5072503.htm?fr=aladdin

Baike[EB/OL].http://baike.baidu.com/view/401141. $\underline{\mathrm{htm}}$

Baike[EB/OL].http://baike.baidu.com/view/336793 $\underline{6 . h t m}$

\section{References from Other Literature:}

QIAN Li-li. Peer-to-peer Communication Protocol and its Security [J]. Net Security Technologies and Application, 2003(7):49-54.

WEI Chang-fa. Research and Implementation of Strategies for Security Peer-to-Peer of Instant Messaging [D]. Changsha:Hunan University,2007.

WU Xiao-mao. Discussion of Security Communication Protocol Model Based on

Peer-to-Peer Instant Information Exchange [J]. Netinfo Security, 2012(4):71-74, 80.

LI Yuan-chen,LIU Wei-qun. Point-to-Point Communication Based on TCP/IP Protocol [J].Journal of Luoyang Teachers College, 2002, 21(2):61-64.

LU Tianbo, FANG Binxin, SUN Yuzhon. etc. Performance Analysis of a Peer-to-Peer Anonymous Communication Protocol WonGoo [J].Computer Engineering, 2006, 32(2)26-28,155.

HUANG Huan, HUANG Zan. A Point-to-point Communication Method Based on TCP Protocol

[J].Computer and Modernization, 2005(9):62 64. 